\title{
A step-by-step translation of evidence into a psychosocial intervention for everyday activities in dementia: A focus group study
}

\author{
Clarissa M. Giebel ${ }^{1,2,}{ }^{*}$, David Challis ${ }^{1}$, Nigel M. Hooper ${ }^{3}$, Sally Ferris ${ }^{4}$ \\ ${ }^{1}$ Personal Social Services Research Unit, The University of Manchester, Manchester, UK \\ 2 School of Psychological Sciences, The University of Manchester, Manchester, UK \\ ${ }^{3}$ Institute of Brain, Behaviour and Mental Health, The University of Manchester, Manchester, UK \\ ${ }^{4}$ Together Dementia Support, Manchester, UK
}

*Correspondence should be addressed to: Clarissa Giebel, PSSRU, $2^{\text {nd }}$ Floor Crawford House, The University of Manchester, Booth Street East, Manchester, M13 9QS, UK. Email: clarissa.giebel@manchester.ac.uk; Tel: +44 1612755652

\section{Acknowledgements}

The authors wish to thank the people with dementia and carers at TogetherDementiaSupport and NHS staff at Manchester Mental Health and Social Care Trust for participating in this study.

\section{Declaration of Conflicting Interests}

The authors declare no conflicts of interest.

\section{Funding}

This article presents independent research supported by the National Institute for Health Research (NIHR), under its Programme Grants for Applied Research programme (Grant Reference Number: DTC-RP-PG-0311-12003), and forms part of the doctoral thesis of CG. This work was also supported by the Manchester Institute for Collaborative Research on Ageing (MICRA) and Dementia@Manchester through the MICRA Seedcorn Funding Award 2015. 


\title{
JOURNAL: Aging \& Mental Health
}

\begin{abstract}
Objective: In order to increase the efficacy of a psychosocial intervention in dementia, a step-by-step process involving the translation of evidence and engaging with the public should be adhered to. This study describes such a process via a two-stage focus group with people with dementia (PwD), informal carers, and healthcare staff working with PwD.

Methods: Based on previous evidence, general aspects of effective interventions were drawn out. These were tested in the first focus groups with informal carers and PwD and with staff. In the second focus groups, both the group with PwD and carers and the staff group were consulted about the detailed components. Feedback from these second groups was used to advance the intervention format further.
\end{abstract}

Findings: Existing evidence and four focus groups helped to identify six practical and situationspecific elements worthy of consideration in planning an intervention, including underlying theory, feasibility, and personal motivations. Carers, PwD, and staff highlighted the importance of rapport between the practitioner and the person with dementia prior to commencing the intervention. It was also considered important that the intervention would be personalised to each individual, by allowing choice of activities to focus on. Assistive technology was also mentioned as a means for those PwD without a carer to aid independent living.

Conclusions: This study shows how valuable public and staff involvement can be to the development of an intervention for dementia, and outlines a suggested process of public involvement for future interventions. The next step would be to formally test these intervention elements.

Keywords: dementia, activities of daily living, translation, intervention 


\section{Introduction}

Deficits in initiating and performing everyday activities are some of the primary symptoms of dementia (Giebel, Burns, \& Challis, 2016; Giovannetti et al., 2015). Both instrumental activities of daily living (IADLs), such as using the telephone, and basic ADLs (ADLs), such as personal hygiene, become impaired from the early stages of dementia onwards, although more complex IADLs deteriorate to a greater extent early on (Giebel et al., 2015; Peres et al., 2008; Pocnet et al., 2013).

Despite everyday functioning being a primary symptom of dementia, there are few cognitive and non-pharmacological interventions that have directly targeted these deficits (Avila et al., 2004; Giebel \& Challis, 2015; Kurz et al., 2009). Avila and colleagues (2004) for example used verbal association techniques, amongst others, to improve specific daily activities over a 14-week period, whilst Kurz et al. (2012) targeted daily routines to measure their effectiveness on global ADL performance in Alzheimer's disease. Those that were identified also indicated mixed evidence of effectiveness (Avila et al., 2004; Dechamps et al., 2011; Kurz et al., 2012). Possibly, because of the range of elements in each intervention, such as carer involvement, frequency of sessions, and integration of underpinning psychological theory, it was difficult to establish which set of components contributed to an effective outcome. One of the most striking revelations about the interventions included was the lack of articulation of the relationship between cognition and everyday functioning. This became apparent from the lack of theoretical background discussed about the interventions, and also by the fact that several studies measured global functioning, and not individual activity performance. With different individual activities being associated with different cognitive functions (Brown et al., 2011; Ramsden et al., 2008), cognitive interventions need to focus on individual activities. If an intervention, for example, attempts to improve executive functioning, overall daily functioning might not be improved to a significant degree. However, targeting individual activities and in this case those activities shown to be reliant on executive functioning will create a clearer foundation for, and greater effect of, an intervention. Hence, future cognitive interventions for IADLs and ADLs should integrate this knowledge better in the intervention development and design process.

One important element in the design process of an intervention, and in research in general, is public involvement. In the guidelines on developing complex interventions, the Medical Research Council (2006) strongly suggests involving potential users in the development process of an intervention, as it can strengthen the development of a feasible intervention. The National Institute for Health Research has highlighted six key values of good public involvement in the values and principles framework (INVOLVE, 2015), which include respect, support, transparency, responsiveness, fairness of opportunity, and accountability. Involving the public in the research design process can help ensure that both the needs, but also the experiences of those potentially involved in such an intervention are taken account of. Underlying this is the importance that an intervention is feasible and addresses issues that would motivate people with dementia and their carers to participate, since such engagement can possibly take up a significant proportion of time. Furthermore, an intervention can benefit from the input of staff who are regularly involved in either sanctioning or providing training to people with dementia on a regular basis. Indeed, public involvement has been shown to help in many areas of research, ranging from the design of studies or systematic reviews to 
aiding recruitment and involving service users in the design of a home support intervention (Backhouse et al., 2016; Burnell et al., 2015; Illiffe et al., 2013; Mockford et al., 2016).

The objective of this study was to outline the process of designing a cognitive/psychosocial intervention for everyday functioning in people with dementia (PwD) through public involvement and focus groups with informal carers and PwD. In a recent focus group study, PwD and their carers have shared their opinions on an established Dutch occupational therapy intervention for adaptation to the UK (Hynes et al., 2016). This latter study confirmed the vital role of patient and carer feedback in the intervention design process, whereas the present study involves PwD, carers, and staff, at a much earlier stage in this process. This study also acts as an exemplar to use public involvement in future intervention design processes.

\section{Method}

\section{Elements of public involvement}

This study involved the public on all six key values of the INVOLVE (2015) framework. Respect - the contributions of carers and staff were recognised in shaping the intervention directly. Support Carers, PwD, and staff could be flexible in their involvement, and it was not required for the same carers and staff to be present at both focus groups. Their expenses were reimbursed. Transparency the research team was open about the expectations of carers and staff in taking part in the focus groups, by highlighting that there were no correct or incorrect opinions, and that participants only were asked to share what they wished to share. Responsiveness - the research team can in future implement the feedback from carers, PwD, and staff into the design of an intervention. Fairness of opportunity - participating in the carer and PwD group was open to any carer and PwD that attended the group service across South Manchester, and to any staff dealing with PwD in their job at the Trust involved. Accountability - the research team was accountable to the participants and vice versa, which meant that the research team openly discussed how their opinions had been integrated into the intervention at the beginning of the second set of focus groups and in a letter after the second set of focus groups were conducted.

\section{Development of an intervention}

This qualitative focus group study comprised five steps in which the intervention was developed. Step I involved synthesising the evidence gathered as part of the researcher's $\mathrm{PhD}$ (i.e. Giebel \& Challis, 2015; Giebel, Challis, \& Montaldi, 2014; 2015; Giebel et al., 2014) into domains that are considered likely to contribute to an intervention's effectiveness.

Step II comprised the first set of focus groups, one with carers and PwD and one with staff. The focus groups were guided by the domains synthesised in the first step. Participants had the opportunity to share their opinions on these domains, and potentially identify additional domains which should be incorporated in an intervention. 
Step III involved the researcher integrating the findings from the first set of focus groups into the development of an intervention. Following on from the specification of the domains, the evidence was synthesised into the individual components of the intervention, thus further clarifying the final intervention.

Step IV involved the second set of focus groups with carers and with staff. In the focus groups, the research team informed the participants how their first contributions had had an impact on the intervention development. Furthermore, the research team sought the participants' advice on the revised intervention components which were specified in Step III.

In the final step, Step V, findings from Step IV were integrated to produce a further, theoretical and evidence-based intervention to maintain or improve everyday functioning in dementia.

\section{Focus groups}

\section{Participants}

The focus groups involved two different types of participants - informal carers and people with dementia participated separately from stakeholders, namely National Health Service (NHS) staff (including occupational therapists, assistant practitioners, dementia support workers, speech and language therapists, social workers, and community research nurses) who were involved in the diagnosis and management of dementia in their day-to-day work. Carers and PwD were recruited via a support group organisation in South Manchester, and staff were recruited via a large NHS Mental Health Trust in Manchester. Ethical approval was obtained via proportionate review from the Yorkshire and the Humber - Leeds East Research Ethics Committee (16/YH/0070).

At the beginning of each focus group, written informed consent was obtained and the focus groups were audio recorded and transcribed (CG). Focus groups were conducted between April and June 2016 on the premises of the carer group organisation or on NHS premises, and lasted between 45 and 60 minutes.

\section{Procedure}

As noted, the development process comprised five stages. First, the team extracted information from two literature reviews in the field and three studies, forming the foundation of the evidence for the intervention development (Giebel \& Challis, 2015; Giebel, Challis, \& Montaldi, 2014; 2015; Giebel et al., 2014; 2015). In this step, the team specifically extracted those domains considered important generally for effective psychosocial and cognitive interventions in the field of IADL functioning. Second, the first set of focus groups, one with carers and one with staff, were used to obtain data on those aspects considered important by these two groups in an intervention for people to participate in and for the intervention to be effective. In the third step, this information was integrated into improvement of the intervention design, giving more specific focus upon daily activities. Fourth, these enhanced specifications of the intervention and its components were discussed in the second set of 
focus groups. This revised information was synthesised in the fifth step of the design process to finalise those elements considered vital in an everyday functioning intervention for dementia.

\section{Data analysis}

Data were analysed using both a deductive approach based on the specified identified elements of an intervention for everyday functioning (Figure 1) and through an inductive thematic analysis approach (Braun \& Clarke, 2006). Transcripts were read several times and coded to generate themes that were either already part of the identified elements, or were novel additions to consider.

\section{Results}

The focus groups comprised between five and eleven participants, and a total of three PwD, 15 carers, and 12 staff participated across the four groups. The majority of informal carers, all PwD and staff were female, whilst two male carers participated in the focus groups. We did not collect any data on age or other demographics. The majority of participants in the PwD and carer, and in the staff groups varied between the first and second focus group.

\section{Step I: Elements of interventions}

The first step of this process involved synthesising evidence into the individual elements of the intervention. In doing so, six elements, four practical and two situation-specific ones, emerged. First, the length and frequency of training/intervention sessions emerged as one of the deciding elements of the efficacy of an intervention. One recent review showed that cognitive interventions for everyday activities in mild dementia and $\mathrm{MCl}$ varied regarding their length (for example three weeks or three months) and their intensity (one session a week or three sessions a week) (Giebel \& Challis, 2015). Although the number of studies found in this field were rather small $(\mathrm{N}=14)$, comparison between studies appeared to suggest that both the length and frequency of intervention sessions contributed to the success of interventions. In particular, a 12-week intervention with one-weekly training sessions was not successful in improving everyday functioning (Kurz et al., 2012), whilst other programmes only lasting one or three weeks (Dechamps et al., 2011; Zanetti et al., 1997) with higher training frequency showed significant improvements.

Second, the limited number of interventions in this field have primarily targeted global functioning, and solely performance. However, this may be one of the reasons for some of the interventions lack of effectiveness. Hence, a further critical component appeared to be focusing on individual activities, and targeting both initiative and performance of an activity. Several studies have indicated varying levels of deficits in initiating and performing an activity in dementia (Teunisse et al., 1997; Giebel et al., 2014; submitted; Voigt-Radloff et al., 2012). Moreover, a recent evaluation found that some activities had a significantly better preserved performance than initiative, and vice versa for other activities, which are also found to vary between dementia subtypes (Giebel et al., 2016). Hence, 
focusing solely on the performance of activities could possibly reduce the efficacy of an intervention, so that strategies would also need to be developed to raise initiative levels.

Third, the level of knowledge about the cognitive underpinnings of individual activities should contribute to the selection process of the targeted interventions. The greater the understanding of an activity, the better we can develop an evidence-based intervention by targeting those areas of cognition which are impaired and contributing to the poor IADL performance. This is a fluid aspect however, as new knowledge is accumulating and thus the selected activities could change.

Fourth, feasibility is also relevant when selecting suitable activities. For example, implementing an intervention for improving or maintaining driving is less feasible, as well as ethically and legally difficult to implement, than an intervention to maintain preparing a hot meal. Focusing on computer use, for example, might also not be feasible, as some people never have been able to use the computer or do not own one. Activities involving outdoor elements might also be considered not feasible, such as going shopping, following familiar routes, or taking public transport. Carers might feel uncomfortable if the person conducting the intervention were taking the PwD outside, unless the carer were also present.

Fifth, the intrinsic motivation of participating in an intervention needs to be present, which is related to raising initiative of engaging in IADLs. This is a situation-specific element of an intervention and the motivation can be strengthened by focusing an intervention on those aspects of daily life which are either found to be most impaired or to be affecting the well-being of the PwD or the carer the most. To elaborate, if, for example, dressing for the PwD takes up very little time during the day, this activity might not affect the carer's well-being to a great extent.

Sixth, interventions may be differentially effective depending on the presence, or the level of involvement, of the carer. With the presence of a carer, techniques taught by the person conducting the intervention, such as an occupational therapist or trained nurse, can be implemented throughout the intervention more effectively with the carer reminding the PwD. Indeed, the many interventions for dementia in the home environment are conducted with dyads of PwD and carers (Gitlin et al., 2010; Graff et al., 2007; Samus et al., 2014). However, some PwD may not have a relative who can care for them, so that it is important that interventions are adapted wherever possible so as to not exclude those PwD.

Figure 1 captures those six elements which appeared to need consideration in psychosocial interventions for everyday functioning.

\section{[insert here Figure 1]}

\section{Step II and III: First set of focus groups and implementation of feedback}

\section{Continuity and Rapport}

Carers raised several issues they considered important to take account of when developing a cognitive intervention for the purpose of improving everyday functioning. Carers considered it 
important that the same person came to visit the PwD throughout the intervention, and that this professional also knew something about the PwD's history in order to better relate to the person:

"I think more of a friend than a stranger, that can relate to somebody in the past." (Carer)

This was corroborated by staff, who clearly highlighted the need for trust between the person conducting the intervention and the beneficiary:

"I think as professionals generally we build up trust before we would sort of intervene in any sort of way." (Staff)

\section{Length and frequency}

Carers also shared their opinions on the frequency of such an intervention. One carer suggested for the PwD to receive training, followed by a break, followed by another set of training weeks, whereas other carers suggested that any intervention needs to happen more than once a week, as otherwise the learning would be compromised and forgotten:

"I think it's quite difficult, but I would suggest, I mean, I'm talking from my own personal experiences that my aunt has been to a falls clinics a few times, but she's been, you know, two or three times, I think perhaps a short period and then a break and then another short period and a break and then another short period of the same thing. "Cause she does remember how to sit in a chair now." (Carer) However, staff highlighted that the frequency and intensity of such an intervention would strongly depend upon the individual:

"It is varied, depending on someone's level of function, and how difficult that person might be. That's

where I've been this morning, takes half an hour before he calms down and lets you observe or anything like that, so it can be quite long-winded. And then you could be there for ages. Other people that will cooperate straight away and they are motivated." (Staff)

\section{Personal Motivation}

On being asked how carers would select those activities that should be addressed in an intervention, carers and staff stated that this had to be considered on an individual basis, since whereas PwD might struggle with for example preparing a hot drink or washing, others might experience greater difficulty more with preparing a meal. Of course, activities might be selected not only due to the type of impairment, but also due to their impact on the carers:

"It's stressful, you know, because you are standing at the checkout and trying to put things on the ... belt and then I say 'you take them out the trolley and put them on and I can start packing', then turn around and she's taken them off and back into the trolley and I say 'Right, it's OK, I'll do it all', and get to the you know, and get to start packing and people there, 'cause she stood there, are looking at her. You can see them thinking 'Why is she just stood there?' and she's got all this shopping to try and put it on there, start packing.. You know, that thing is really quite irritating." (Carer) 
"I found it difficult because they said I had to do three, but I couldn't manage to, because I can't multitask any more. So I was only able to do the one." (PwD)

"It should be really what the client wants, and what they really need to be able to do. That's where I would start from. And usually with cognitive rehab techniques, they pick about two or three goals, and then you just work on those goals, but they are chosen by the client. So it might be learning how to use the remote so they can work the TV, or learn how to use the mobile phone, or it might be making a brew." (Staff)

\section{Living without a carer}

Some people with dementia might not have a family carer or not live with them. In those situations, it seemed important to adapt an intervention so as to not rely on carers to perform parts of it. Assistive technology was considered a vital element, whilst staff were also wary of some older people not wanting or being able to learn to use devices such as tablet devices:

"I don't know whether it was on Panorama or so last week, but there was a doctor who's got dementia, and she wanted to make a cup of tea, and she used the tablet. Did anyone watch that? ... So I think, those who are OK with maybe like a tablet or having a little device that has Step 1 shows a picture of getting the cup; Step 2 putting the cup down or whatever or put the milk in, and all of that. So assistive technology again. And like the medication dispensers, or different things like that phone the man was talking about with the GPS." (Staff)

One finding that emerged during the consultations was that carers in general had little knowledge of what an intervention comprises. This was because some carers appeared to have received little support or guidance post-diagnosis, which was a source of distress.

Step II thus highlighted several areas which should be considered when designing an intervention, including continuity and rapport, length and frequency, personal motivation, and the presence or absence of a carer. The majority of these elements had also emerged from the evidence base in Step I, and thus corroborated Step I. Step III involved developing some more intricate questions about non-pharmacological interventions in this field of dementia, which were posed in the two focus groups of Step IV.

\section{Step IV and V: Second set of focus groups and implementation of feedback}

After the learning from the first focus groups had been integrated into the intervention development, carers and staff were consulted a second time about more intricate details of the planned intervention. With both groups having mentioned the importance of building up rapport prior to the intervention, in the second round both groups felt that the person conducting the intervention should visit a few times in the home environment, to make the PwD feel comfortable. Staff also stated that it might be useful for the professional to accompany another professional who is already familiar to the PwD: 
"It's better in your own home, because there is not a lot of distractions. You are familiar with it, you are comfortable with it. It's your home, and if you don't want them there, you can tell them to go, you know. You feel more in control in your own home, so, I think that's better. And also, they'd have to come a few times quite closely together. Get it to a habit. Not many times, but two or three times, before people would be accepted in the home situation, and therefore trusted I think. Going out and meeting new people is just very confusing." (Carer)

In addition to building up rapport, carers also stated in the first meeting that they would also value receiving some direct benefit from the intervention. In particular, carers thought a regular carer support group would be welcome, which should not only serve as a means of social interaction with other carers, but also as a learning platform offering education and coping strategies:

"I think when you are talking about coping and people to deal with behavioural problems and so on and so forth, information about the disease as well, if you can give, at that point, would be very welcome. Not just how to give it up, why need to do it that way, but you know, how it's affecting the behaviour." (Carer)

A support group could act as one means of motivation for carers to participate in the intervention. To stay motivated, staff reported that if PwD were personally motivated from the start to perform an activity again, provided they had the insight, the motivation would remain. In addition to this, the professional conducting the intervention could provide weekly feedback to remind the PwD of their small but positive achievements:

"Maybe with once a week, I'm thinking, having a discussion or feedback session to find some of the positive things of what they are doing. They might not be improving, but they might be staying the same for example. So it's trying to find the positive if there isn't an easy positive. Positive kind of feedback, that would motivate some people." (Staff)

\section{Discussion}

This public involvement study has contributed positively to the design process of cognitive interventions for everyday functioning in dementia. It also offers an exemplar structure for future public involvement and intervention development in this area. Involving service users and those that are frequently involved in the active management of such interventions is indeed recommended by the Medical Research Council (2006), for research to truly capture the needs and wishes of those affected by the condition. Considering that most carers had no understanding of interventions prior to the focus groups, it would seem even more vital that carers are informed about possible nonpharmacological forms of treatment post-diagnosis, whilst simultaneously being consulted about their experiences.

Based on existing evidence and on data collected from the focus groups, it emerged that a psychosocial intervention aiming at improving everyday functioning in dementia should consider both 
practical and situation-specific elements. On a practical side, carers and staff could not state a 'golden standard' for the length or frequency of an intervention, but suggested that it was important for the practitioner visits to be relatively close together, as the PwD might otherwise easily forget things learned in the previous session. In order for a practitioner to be allowed into the home on a regular basis however, both groups stated the importance of developing good rapport between the practitioner and the PwD. One method to build up rapport and develop familiarity with the PwD might be to do life story work in a few sessions prior to the actual intervention. Life story work is increasingly used in dementia practice, and allows a practitioner to find out about the PwD's life story, writing this down jointly, and using this in the care process (McKeown et al., 2015). Life story work has been found to have positive effects on the care of the PwD (Gibson \& Carson, 2010) and is recommended in national dementia guidelines (DH, 2009; NICE, 2006). In a similar focus group study by Hynes and colleagues (2016) on an occupational therapy intervention for people with dementia and their carers, continuity of support also constituted an important element of an effective intervention. This was not covered in the present focus groups, but should also be considered in the intervention design process to truly capture the needs and wishes of those affected by dementia.

Another practical aspect for the intervention would be to pre-select a number of daily activities for focus, based on existing theory and knowledge about their cognitive underpinnings (practical element). As staff stated, PwD can probably only be trained in two or three specific activities in an intervention. Thus, taking into account which activities would be most feasible and possessing most evidence in terms of underpinning theory, it appears that pre-selecting some activities would be an important step prior to approaching participants about their intrinsic motivation. This selection process should be based on the feasibility of an activity, with for example driving perhaps being difficult to improve taking into account the safety of other drivers. Additionally, the selection process should take into account the available evidence into the link between cognition and everyday functioning, as this could help develop specific cognitive tasks for training. Although the evidence base is very limited in this field, for example some research suggests that short-term memory is associated with finance management (Brown et al., 2011). Whilst improving short-term memory in dementia has been little explored in dementia to date, by for example chunking material which improved verbal working memory in Alzheimer's disease (Huntley et al., 2016), yet positive effects on trained tasks in healthy older adults (Zinke et al., 2014), this connection between finance management and short-term memory might suggest that training in this activity should focus upon improving this form of memory. Alternatively, a solution might be to adapt the activity or to use an aid, which depends on a detailed analysis of an activity.

One of the most important findings of this public involvement exercise was that such an intervention needs to be malleable in order to suit each individual PwD. Pre-selection of certain everyday activities offers a good platform for people to choose, based on their own needs and situation. This would increase personal motivation of participants which is required for people to take part over a prolonged period of time. In order to maintain high levels of motivation and reduce dropout rates throughout, motivation could be sustained by highlighting the positives of the impact of training on a frequent basis in consultation with the PwD. This might be conducted face-to-face, or via 
technology such as telephone calls or skype, depending on which was more suitable to the participant. With the level of adherence being significantly linked to the effectiveness of an intervention (Inouye et al., 2003), treatments clearly need to be personalised to both the PwD and the family carer.

Another situation-specific component in the development process is the need for adaptation, depending on the presence or absence of an informal carer. In those circumstances where a carer is unavailable, the intervention should more strongly rely on assistive technology and technology-based apps on tablets. However, the level of familiarity with technology of the PwD needs to be taken into account since some PwD may never have used a tablet or smartphone previously. Assistive technology is frequently used in dementia care (Gibson et al., 2016), but it could be that technologies from other cognitively impaired populations (Gillespie et al., 2012) might be employed as part of such an intervention for PwD living on their own. This level of personalisation of an intervention, focusing on both the motivation and availability of a carer, might be one of the reasons why some previous interventions in this field had little significant effect on everyday functioning (i.e., Kurz et al., 2012).

Some limitations were experienced as part of this process. Although every attempt was made to involve a mix of carers, PwD, and staff, the majority of carers, and all PwD, were female. This is perhaps not surprising considering that the majority of dementia carers are female (ARUK, 2015) and indeed suggests that the groups were representative of the carer population at large. However, there may have been some issues surrounding gender roles of everyday activities, such as activities usually not performed by some male PwD, which were thus not captured. Furthermore, the PwD who took part in the focus groups were only in the second focus group. Whilst this might have resulted in less input in the first few steps of this process, the second focus groups re-iterated elements from the first focus groups, so that PwD did have the opportunity to add their thoughts retrospectively. One further limitation might concern the qualitative design process, in that only one researcher analysed the data. However, findings from the focus groups corroborate findings from the evidence base (Step I), so that this public involvement approach to intervention design process appears to have added novel contributions to a real-life implementation aspect in dementia research. Whilst this type of intervention would predominantly involve occupational therapists, a mix of staff groups was included to provide a wider perspective.

\section{Conclusions}

This paper outlines a detailed process of translating evidence into elements of a psychosocial intervention by adhering to the guidelines of intervention development from the Medical Research Council (2006), and closely involving the public as an element of good practice in research design (INVOLVE, 2015). There are practical and more situation-specific/individual elements to such an intervention. It is important to consider existing knowledge, theory, and also feasibility on the practical side, but in addition more individual issues of personal motivation and presence of a carer. Future intervention development could benefit from the structure outlined here, including the advantages of receiving feedback and guidance on the needs, wishes, and abilities of people with dementia, their 
carers, and staff by involving the public in the design process. A next step in the present work is to take forward these elements and implement such an intervention which appeared to be both effective and feasible in a real-life context. 


\section{References}

Alzheimer's Research UK (2015). Women and dementia: A marginalised minority. Cambridge: Alzheimer's Research UK.

Avila, R., Bottino, C.M.C., Carvalho, I.A.M., et al. (2004). Neuropsychological rehabilitation of memory deficits and activities of daily living in patients with Alzheimer's disease: a pilot study. Brazilian Journal of Medicine and Biological Research, 37, 1721-1729.

Backhouse, T., Kenkmann, A., Lane, K., Penhale, B., Poland, F., \& Killett, A. (2016). Older care-home residents as collaborators or advisors in research: a systematic review. Age \& Ageing, doi: 10.1093/ageing/afv201

Braun, V., \& Clarke, V. (2006). Using thematic analysis in psychology. Qualitative Research in Psychology, 3, 77-101.

Brown, P.J., Devanand, D.P., Liu, X., \& Caccappolo, E. (2011). Functional impairment in elderly patients with mild cognitive impairment and mild Alzheimer disease. Archives of General Psychiatry, 68(6), 617-626.

Burnell, K.J., Selwood, A., Sullivan, T., Charlesworth, G.M., Poland, F., \& Orrell, M. (2015). Involving service users in the development of the Support at Home: Interventions to Enhance Life in Dementia Carer Supporter Programme for family carers of people with dementia. Health Expectations, 18(1), 95-110.

Dechamps, A., Fasotti, L., Jungheim, J., Leone, E., Dood, E., Allioux, A., Robert, P.H., et al. (2011). Effects of Different Learning Methods for Instrumental Activities of Daily Living in Patients with Alzheimer's Dementia: A Pilot Study. American Journal of Alzheimer's Disease and other Dementias, 26(4), 273-281.

Department of Health (2005). Research Governance Framework for Health and Social Care. 2nd Edition. London: Department of Health.

Department of Health. (2009). Living well with dementia: a national dementia strategy. London: DH.

Fudge, N., Wolfe, C.D.A., \& McKevitt, C. (2007). Involving older people in health research. Age and Ageing, 36(5), 492-500.

Gibson, F., \& Carson, Y. (2010). Life story work in practice: aiming for enduring change. Journal of Dementia Care, 18, 20-22.

Gibson, G., Newton, L., Pritchard, G., Finch, T., Brittain, K., \& Robinson, L. (2016). The provision of assistive technology products and services for people with dementia in the United Kingdom. Dementia, 15(4), 681-701.

Giebel, C.M., Burns, A., \& Challis, D. (2016). Taking a positive spin: Preserved initiative and performance of everyday activities across mild Alzheimer's, vascular, and mixed dementia. International Journal of Geriatric Psychiatry, doi: 10.1002/gps.4553

Giebel, C., and Challis, D. (2015). Translating cognitive and everyday activity deficits into cognitive interventions in mild dementia and mild cognitive impairment. International Journal of Geriatric Psychiatry, 30, 21-31.

Giebel, C.M., Challis, D., \& Montaldi, D. (2014). A revised Interview for Deterioration in Daily Activities in Dementia (R-IDDD) reveals the relationship between social activities and wellbeing. Dementia, doi: $10.1177 / 1471301214553614$

Giebel, C.M., Challis, D., \& Montaldi, D. (2015). Understanding the Cognitive Underpinnings of Functional Impairments in Early Dementia: A Review. Aging \& Mental Health, 19(10), 859-875.

Giebel, C.M., Sutcliffe, C., Stolt, M., Karlsson, S., Renom-Guiteras, A., Soto, M., Verbeek, H., Zabalegui, A., \& Challis, D. (2014). Deterioration of Basic Activities of Daily Living and their Impact on Quality of Life across different cognitive stages of dementia: a European study. International Psychogeriatrics, 26(8), 1283-1293.

Gillespie, A., Best, C., \& O'Neill, B. (2012). Cognitive Function and Assistive Technology for Cognition: A Systematic Review. Journal of the International Neuropsychological Society, 18, 1-19.

Giovannetti, T., Seligman, S.C., Britnell, P., Brennan, L., \& Libon, D.J. (2015). Differential effects of goal cues on everyday action errors in Alzheimer's disease versus Parkinson's disease dementia. Neuropsychology, 29(4), 592-602.

Gitlin, L.N., Winter, L., Dennis, M.P., Hodgson, N. and Hauck, W.W. (2010a). A biobehavioral homebased intervention and the well-being of patients with dementia and their caregivers: the COPE randomized trial. Journal of the American Medical Association, 304, 983-991.

Graff, M.J., Vernooij-Dassen, M.J., Thijssen, M., Dekker, J., Hoefnagels, W.H. and Olderikkert, M.G. (2007). Effects of community occupational therapy on quality of life, mood, and health status in dementia patients and their caregivers: a randomized controlled trial. Journals of Gerontology Series A: Biological Sciences and Medical Sciences, 62, 1002-1009. 
Huntley, J.D., Hampshire, A., Bor, D., Owen, A., and Howard, R.J. (2016). Adaptive working memory strategy in early Alzheimer's disease: randomized controlled trial. The British Journal of Psychiatry, DOI: 10.1192/bjp.bp.116.182048

Hynes, S.M., Field, B., Ledgerd, R., Swinson, T., Wenborn, J., di Bona, L., Moniz-Cook, E., Poland, F., \& Orrell, M. (2016). Exploring the need for a new UK occupational therapy intervention for people with dementia and family carers: Community Occupational Therapy in Dementia (COTiD). A focus group study. Aging \& Mental Health, 20(7), 762-769.

Illiffe, S., McGrath, T., \& Mitchell, D. (2011). The impact of patient and public involvement in the work of the Dementias \& Neurodegenerative Diseases Research network (DeNDRoN): case studies. Health Expectations, 16(4), 351-361.

Inouye, S.K., Bogardus, S.T., Williams, C.S., Leo-Summers, L., \& Agostini, J.V. (2003). The Role of Adherence on the Effectiveness of Nonpharmacologic Interventions: evidence from the Delirium Prevention Trial. Journal of the American Medical Association, 163(8), 958-964.

INVOLVE (2015). Public involvement in research: values and principles framework. INVOLVE: Eastleigh.

Kurz, A., Pohl, C., Ramsenthaler, M., \& Sorg, C. (2009). Cognitive rehabilitation in patients with mild cognitive impairment. International Journal of Geriatric Psychiatry, 24, 163-168.

Kurz, A., Thoene-Otto, A., Cramer, B., Egert, S., Froelich, L., Gertz, H.J., et al. (2012). CORDIAL: Cognitive Rehabilitation and Cognitive-behavioral Treatment for Early Dementia in Alzheimer Disease: A Multicenter, Randomized, Controlled Trial. Alzheimer Disease \& Associated Disorders, 26(3), 246-253.

McKeown, J, Ryan, T., Ingleton, C., \& Clarke, A. (2015). 'You have to be mindful of whose story it is': The challenges of undertaking life story work with people with dementia and their family carers. Dementia, 14(2), 238-256.

Medical Research Council (2006). Developing and evaluating complex interventions: new guidance. London: Medical Research Council.

Mockford, C., Murray, M., Seers, K., Oyebode, J., Grant, R., Boex, S., Staniszewska, S., Diment, Y., Leach, J., Sharma, U., Clarke, R., \& Suleman, R. (2016). A SHARED study - the benefits and costs of setting up a health research study involving lay co-researchers and how we overcame the challenges. Research Involvement and Engagement, 2, 8-19.

National Collaborating Centre for Mental Health (2006). A NICE-SCIE guideline on supporting people with dementia and their carers in health and social care. National Clinical Practice Guideline Number 42. London: British Psychological Society and Gaskell.

Peres, K., Helmer, C., Amieva, H., Orgogozo, J.M., Rouch, I., Dartigues, J.F., \& Barberger-Gateau, P. (2008). Natural History of Decline in Instrumental Activities of Daily Living Performance over the 10 Years Preceding the Clinical Diagnosis of Dementia: A prospective Population-Based Study. Journal of the American Geriatrics Society, 56(1), 37-44.

Ramsden, C.M., Kinsella, G.J., Ong, B., \& Storey, E. (2008). Performance of everyday actions in mild Alzheimer's disease. Neuropsychology, 22(1), 17-26.

Samus, Q.M. et al. (2014). A multidimensional home-based care coordination intervention for elders with memory disorders: the Maximizing Independence at Home (MIND) pilot randomized trial. Am J Geriat Psychiatry, 22(4), 398-414.

Zanetti, O., Binetti, G., Magni, E., Rozzini, L., Bianchetti, A., \& Trabucchi, M. (1997). Procedural memory stimulation in Alzheimer's disease: impact of a training programme. Acta Neurologica Scandinavica, 95(3), 152-157.

Zinke, K., Zeintl, M., Rose, N.S., Putzmann, J., Pydde, A., and Kliegel, M. (2014). Working memory training and transfer in older adults: Effects of age, baseline performance, and training gains. Developmental Psychology, 50(1), 304-315. 
Figure 1. Practical and situation-specific elements of an intervention for everyday functioning

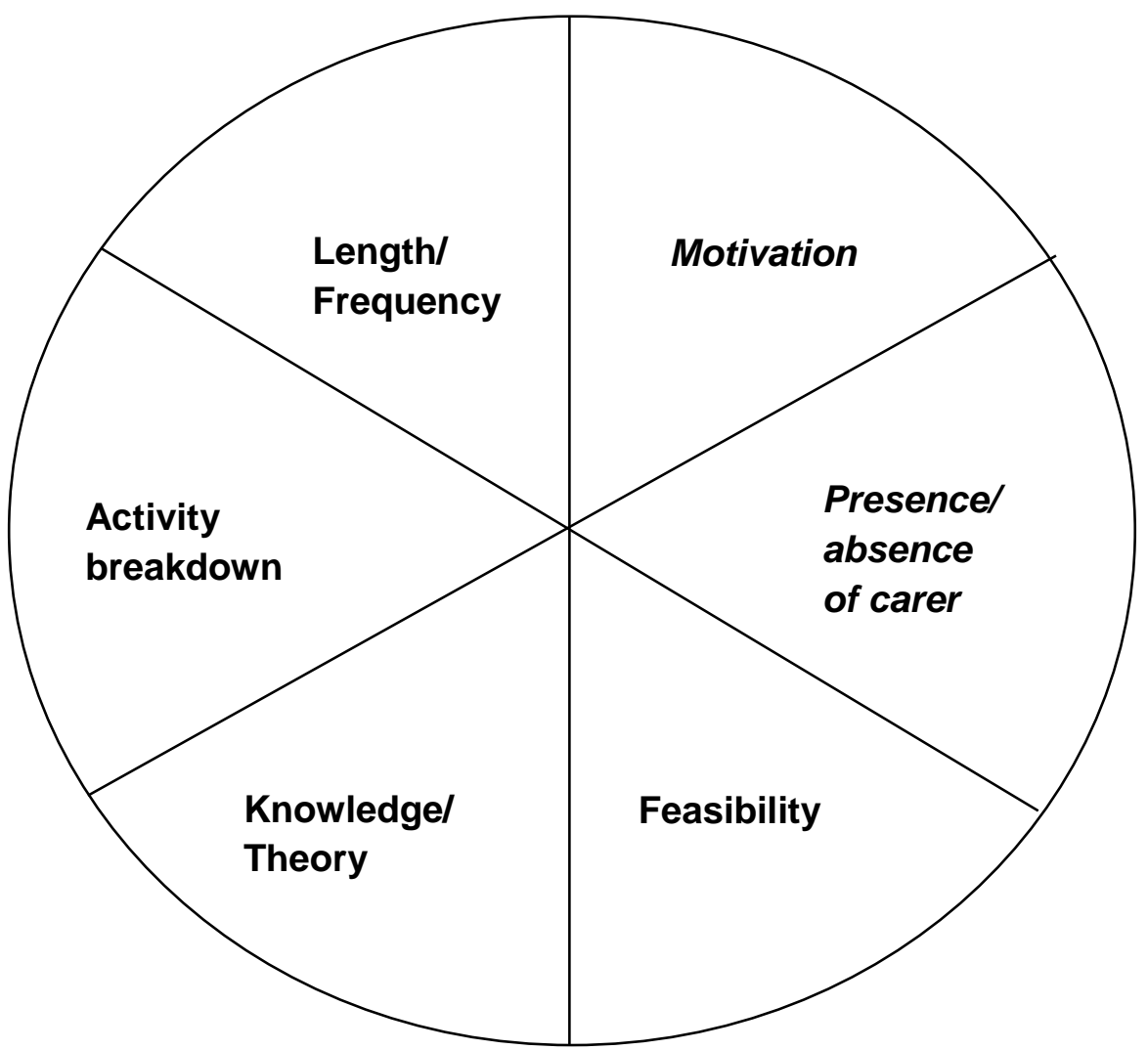

Practical elements of an intervention are in bold, and situation-specific elements are in bold italics. 